\title{
Subtypes of m6A RNA Modification Defines Tumor Microenvironment, Immunotherapy and Pharmacogenomic Landscape in Prostate Cancer
}

\section{Song Li}

Henan University https://orcid.org/0000-0001-7679-1548

\section{Yifan Hou}

Henan University

Junqing Hou

Henan University

Xiaodong Li

Henan University

Jianhua Zhang

Henan University

\section{Shangren Wang}

Tianjin Medical University First Clinical College: Tianjin Medical University General Hospital

Xiaoqiang Liu ( $\sim$ Liuxiaoqiang121021@163.com )

Tianjin Medical University First Clinical College: Tianjin Medical University General Hospital

Primary research

Keywords: RNA modification, prostate cancer, microenvironment, immunotherapy, prognostic signature

Posted Date: August 25th, 2021

DOl: https://doi.org/10.21203/rs.3.rs-815471/v1

License: (9) (i) This work is licensed under a Creative Commons Attribution 4.0 International License. Read Full License 


\section{Abstract}

Background:M6A methylation is an epigenetic modification of RNA molecules, which is potentially related to the occurrence and development of tumors.

Methods:We systematically described the mRNA expression and gene changes of 20 RNA modified m6A related genes in prostate cancer(PCA), and evaluated their expression modification patterns in TCGA database samples. Two groups of clustering samples were used to find 3022 differentially expressed genes (DEGs) which were used for unsupervised clustering, and then the samples were divided into two groups of expression patterns and the significant prognosis of DEGs were screened. Then, we constructed risk scores based on survival and prognosis to quantify RNA modification related subtypes of the tumor. In addition, we also analyzed the relationship between risk_score groups and characteristics of TME, clinical pathology, drug response and the efficacy of immunotherapy.

Results:We demonstrated that the prognostic risk stratification of m6A RNA modification was associated with survival, TME immune cell infiltration and immunotherapy response in patients with prostate cancer. We constructed a risk_score model based on the prognosis of $\mathrm{m6A}$, which was divided into high-risk group and low-risk group. The risk_score-high group was positively correlated with Itreg cells, and the infiltration of $\mathrm{B}$ cells, $\mathrm{T}$ cells, $\mathrm{CD} 4^{+}$, endothelial cells and $\mathrm{T}$ cells $\mathrm{CD} 8^{+}$was higher in risk_score-high group. The gene expression of each immune checkpoint was high in risk_score-high group which had higher TIDE score and MSI score than risk_score-low group.

Conclusions:We conducted a comprehensive analysis and risk stratification of m6A in prostate cancer for the first time, revealed the potential functions of risk stratification in TME, immunotherapy and transcription, and determined their potential therapeutic value in targeted therapy and immunotherapy.

\section{Introduction}

Prostate cancer $(\mathrm{PCa})$ is one of the most common malignant tumors in males and its treatment and prognosis mainly depend on early diagnosis of [1]. Radical surgery and castration are the main treatment methods for early cancer patients, but some patients still have recurrence, metastasis and progression to castration resistant prostate cancer [2]. At present, there is no effective treatment for castration resistant prostate cancer, so exploring new mechanisms and effective treatment methods has been a difficult problem for urology academia. Recent studies have found that m6A is a very common modification in mRNA and LncRNA, which affects RNA splicing, translation, stability and epigenetic effects of some ncRNAs $[3,4]$. The abnormal regulation of m6A modification plays an important role in many types of tumors by affecting the expression of tumor related genes, and M6A modification can promote and inhibit the occurrence and development of tumors [5]. First of all, this is due to the diversity of target genes modified by $\mathrm{m} 6 \mathrm{~A}$, which can be either oncogene or tumor suppressor gene. Secondly, the difference of "writer" or "eraser" involved in the m6A regulatory pathway will have different effects on the increase or decrease of the level of m6A. In addition, the modified m6A can affect its target mRNA by recruiting 
different "readers", which play different roles in tumor development. Further studies on the differential expression and modification mechanism of m6A modification in different tumors will help to reveal the mechanism of m6A in cancer.

The main mechanism of immune checkpoint inhibitors is to activate the body's immune system to control tumor growth, which has been widely used in a variety of malignant tumors [6,7]. Immune checkpoints mainly included cytotoxic T-lymphocyte antigen 4 (CTLA-4), programmed death 1/ programmed death ligand 1 (PD-1/PD-L1), lymphocyte activation gene 3 (LAG-3) [8,9]. Tumor microenvironment (TME) includes microvessels, fibroblasts, stromal cells, immune cells, and cytokines, chemokines, and chemical factors secreted by various cells which form a complex network to regulate the occurrence, development and metastasis of tumor cells. Among them, immune cells play an important role in tumor progression. Antibodies against CTLA4 or PD1/PDL1 have been proven effective in many tumors including renal cell carcinoma, bladder cancer, melanoma, lung cancer and so on [10, 11], indicating that the targeted therapy for tumor microenvironment is feasible. However, it seems that the effect of traditional immunotherapy on these two kinds of immune checkpoint inhibitors in prostate cancer is not obvious [12,13]. Therefore, we need to find out the reasons and further explore new immune checkpoint inhibitors. Some studies have shown that the change of the overall methylation level of m6A is related to the development of cancer, and may be related to the change of a variety of immune cells. The change of methylation level of m6A can be used as an indicator to identify the transformation between different stages of cancer and different subtypes of immune cells [14].

We explored the genetic changes of RNA modified m6A at the genomic level and the interaction of RNA modified m6A at the transcriptome level. We constructed a risk model according to the prognosis related $D R G$, and focused on the application value of risk model in tumor immune microenvironment and immunotherapy. The risk_ score model can guide clinical prognosis and predict patients' response to ICB (Immune Checkpoint Blockade) treatment.

\section{Materials And Methods}

\subsection{Genome and transcriptome analysis of m6A modification}

Cbioportal cancer genomics [15] database is mainly used to solve the problem of large amount of data obtained by large sample tumor genomics research. Through oncoprint function of cbioportal cancer genomics, we can obtain the mutation data information about the genes related to the modification of $\mathrm{m} 6 \mathrm{~A}$, Through the survival option function of cbioportal cancer genomics, we obtained the mutation prognosis analysis data of the genes related to the modification of m6A.The somatic copy number and gene expression data of m6A modification related genes were collected from TCGA database, and the samples were divided into three groups (CNV gain, CNV loss, none CNV) according to the situation of CNV.

\subsection{Subtypes based on the modified gene of $\mathrm{m} 6 \mathrm{~A}$}


We obtained the original count and corresponding clinical information of RNA sequencing data of m6A modification related genes in prostate cancer from the Cancer Genome Atlas (TCGA) data. The correlation map of m6A modified genes was drawn by R software package ggstatsplot, and the multi gene correlation map was displayed by $\mathrm{R}$ software package pheatmap. Spearman's correlation analysis is used to describe the correlation between quantitative variables without normal distribution. The $R$ software package consensusclusterplus (v1.54.0) was used for consistency analysis. The clustering heat map was analyzed by $R$ software package pheatmap ( $v 1.0 .12)$. The genes with variance above 0.1 were retained in the gene expression heat map. Limma software package of $R$ software (version: 3.40.2) was used to study the mRNA differential expression. "Adjusted $P<0.05$ and log2 (fold change) $>1$ or log2 (fold change) <-1" was defined as the screening of threshold mRNA differential expression. The clusterprofiler package in $\mathrm{R}$ was used to analyze the go function of potential mRNA and enrich KEGG pathway. $P$ values less than 0.05 were considered statistically significant.

\subsection{Different risk models of RNA modification}

3022 differentially expressed genes were found by using these two groups of clustering samples, by which the sample were divided into two groups (cluster) by unsupervised clustering. The gene expression heat map and volcano map were drawn by limma software package of R software, and the survival differences between the two groups were compared by KM survival analysis. Single factor Cox regression analysis was carried out on the training set data, and the regression coefficient of the best prognosis gene was obtained by multiple Cox proportional hazard regression model. The expression level and coefficient of each gene were combined by linear combination, and the risk score formula was obtained: risk score $=$ coefficient $1 *$ gene 1 expression $+\ldots+$ coefficient $1{ }^{*}$ gene $\mathrm{N}$ expression. At the same time, the distribution of clinical characteristics of risk score in different groups was compared and analyzed by $\mathrm{R}$ software v4.0.3. GSVA method was used to analyze the functions of the two types.

\subsection{Immune microenvironment and immunotherapy response}

The TIMER algorithm is used to observe the difference of immune cells in different groups of samples by the immunedeconv of R software package. SSIGLEC15, TIGIT, CD274, HAVCR2, PDCD1, CTLA4, LAG3 and PDCD1LG2 are transcripts related to immune checkpoint. The expression values of these 8 genes were extracted and the expression of immune checkpoint related genes was observed. Immune checkpoint blockade (ICB) therapy has revolutionized the treatment of human cancer. Based on the expression profile data, the Tumor Immune Dysfunction and Exclusion (TIDE) algorithm is used to predict the response of a single sample or a subtype to immune checkpoint inhibitors[16]. Microsatellite instability (MSI) data are collected from TCGA database. MSI is caused by the defect of mismatch repair (MMR) gene, which is closely related to tumorigenesis and tumor immunotherapy [17]. The above results are implemented by $R$ software package ggplot2 and pheatmap. Using the cancer therapeutics response portal (CTRP) to discover different risks_Score model group matched potential cancer drugs [18].

\section{Results}




\subsection{Mutation and copy number analysis of genes related to m6A modification}

Genetic changes were analyzed by mutation and copy number. In terms of mutation, 39 (8.58\%) of 452 samples in TCGA-PCA data set had RNA modification mutation, among which ZC3H13 had the highest mutation rate, reaching $21 \%$ (Fig. $1 \mathrm{~A}$ ). In order to explore whether the genetic change of m6A affects the progression of prostate cancer, all samples were divided into two groups according to whether the mutation occurred. The survival analysis of these two groups showed that the survival difference between the two groups was significant $(P=0.021)$, and the prognosis of the mutation group was poor, which indicated that the mutation of m6A gene could affect prostate cancer (Fig.1B). GSVA showed that the mutation group of m6A gene was more concentrated in the cancer hallmark gene set, such as RTK and EASMAPK signaling pathways. This mutation of m6A gene may lead to functional changes, which may affect the prognosis of prostate cancer (Fig.1C).

Copy number amplification generally corresponds to increased expression, while copy number deletion generally corresponds to decreased expression. To further explore whether somatic copy number changes affect the prognosis and progression of prostate cancer, our study showed that the change of copy number of each kind of m6A(Fig.2A), and the expression of each kind of m6A in tumor and paired normal(Fig.2B). It was found that the high frequency of CNV gain of RBM15B, HNRNPC and YTHDF3 in PCA corresponded to the increased expression, which suggested that CNV might be a regulator of the expression of m6A mRNA. However, the CNV loss of some m6A modified genes corresponded to increased expression. The samples were divided into three groups (CNV gain, CNV loss, none CNV) according to the CNV of m6A modified gene. The expression of FTO,YTHDF3, YTHDC2, ZC3H13 and IGF2BP2 in CNV loss group was significantly lower than that in normal group (Fig.2C).

\subsection{Different RNA modification patterns related to hallmark in cancer}

The original count and clinical information of RNA sequencing data of m6A modification related genes were obtained from the Cancer Genome Atlas (TCGA) data set. In the present study, a total number of 452 PCA patients were included from TCGA. The clinicopathologic characteristics of the patients are listed in Table 1. In order to explore different RNA modification patterns, correlation analysis was conducted on the expression data of $20 \mathrm{~m} 6 \mathrm{~A}$ modified genes, and it was found that there was interaction between different types of m6A modified genes (Fig.3A). Next, all samples were clustered according to the expression of these 20 m6A modified genes, and two clusters were obtained (Cluster_1 and cluster_2)(Fig 3B, 3C). Survival analysis showed that there was a significant difference in the prognosis between the two groups (Fig.3D). So what do these two types affect PCA? Two clustering gene expression heat maps and volcano maps (Fig 3E, 3F) were drawn. Go and KEGG methods were used to analyze the two types of differential genes. The $\mathrm{GO}$ of up-regulated gene was mainly enriched in stone modification and covalent chromotin modification, and the KEGG of up-regulated gene was mainly enriched in proteoglycans in cancer and focal adhesion. The GO of down_regulated genes was mainly enriched in oxidative phosphorylation, while KEGG of down-regulated genes was mainly enriched in thermogenesis and oxidative phosphorylation (Fig.3G). 


\subsection{Construct the signature of m6A RNA modification}

Firstly, PCA transcription samples were divided into two groups by using 20 m6A modifying genes and clinical information(Cluster_1 and cluster_2). Using these two groups of samples, 3022 differentially expressed genes (DEGs) were screened, and then the samples were divided into two groups by unsupervised clustering (gene.cluster_A and gene.Cluster_B) , and the heat maps and volcano maps(Fig.4A, 4B) were drawn. Finally, it was found that the samples in gene.cluster_A and Cluster_1 were consistent, and the samples in gene.cluster_B and Cluster_2 were consistent, and the prognosis of gene.cluster_A group was worse than that of gene.cluster_B group (Fig.4C), which also verified that the 3022 DEGs could represent the m6A RNA modification pattern. Univariate analysis of prognosis (Fig.4D) was performed for 3022 differentially expressed genes, and a scoring model (Risk_Score) was constructed using genes with prognostic value, which was used to quantify RNA modification patterns in prostate cancer patients. The risk score of cluster_1 was significantly higher than that of cluster_2 (Fig.4E), and the risk score of high-grade ( $\mathrm{T}$ and $\mathrm{N}$ ) tumors was higher than that of low-grade (Fig.4F, 4G). The PFS survival of risk_score-low group was higher than that in risk_score-high group (Fig. $4 \mathrm{H})$. In order to test whether the risk_score can be used as an independent prognostic indicator, multivariate Cox risk regression was conducted and it was found that the risk_score group is a poor and independent prognostic indicator (Fig.4I). The cancer related pathways of GSVA showed that risk_score-high group was negatively correlated with Cellcycle, EMT and hormone Er, and positively correlated with RTK, EMT and RasMAPK. On the contrary, risk_score-low was negatively correlated with RTK, RasMAPK and EMT, and positively correlated with hormone ER and dnadamage (Fig.4J). Immunocorrelation of GSVA analysis showed that high risk scores were positively correlated with itreg cells, but positively correlated with $\mathrm{B}$ cell, monocyte and $\mathrm{CD} 8^{+} \mathrm{T}$ were negatively correlated; On the contrary, low risk scores were associated with $\mathrm{B}$ cell, macrophage, monocyte and $\mathrm{CD} 8^{+}$, and negatively correlated with Treg cells (Fig.4K).

\subsection{Prediction of immune microenvironment and immunotherapy response by risk_score group}

A large number of articles have reported that immune checkpoint genes and infiltrating immune cells are related to the modification of m6A RNA. Heat map of immune checkpoint gene expression showed that immune checkpoint gene was highly expressed in high risk_score group (Fig.5A). Among them, SSIGLEC15,TIGIT, CD274, HAVCR2, LAG3 and PDCD1LG2 were highly expressed in high risk_score group (Fig. 5B). CIBERSORT method was used to get the proportion of various kinds of cells in each sample, and we found that m6A RNA modification may be strongly related to the invasion of TME cells. According to the high and low score classification, differential expression analysis of the obtained cell data showed that the infiltration of $\mathrm{B}$ cells, $\mathrm{T}$ cells $\mathrm{CD} 4^{+}$, endothelial cells and $\mathrm{T}$ cells $\mathrm{CD} 8^{+}$was higher in risk_scorehigh group (Fig. $5 \mathrm{C}$ ), which may be the reason for its better prognosis. The result of patients' response to ICB treatment predicted by risk_score showed that risk score was positively correlated with TIDE score (Fig.5D), and the TIDE score of high risk group was higher than that of low risk group (Fig.5E). In addition, MSI was closely related to tumor occurrence and tumor immunotherapy, and the MSI score of high risk group was significantly higher than that of low risk group (Fig.5F). Combined with the lower TME of B 
cells, $\mathrm{T}$ cells $\mathrm{CD} 4^{+}$and $\mathrm{T}$ cells $\mathrm{CD} 8^{+}$in the high risk group, we can conclude that the risk_score model can well reflect the tumor immune infiltration pattern and guide the immunotherapy. In order to evaluate the role of risk score in drug response, the relationship between risk score and drug response of cancer cell lines was explored. By using Spearman correlation coefficient, the combination of drug sensitivity between risk score and CTRP database was identified, and 30 kinds of drugs related to risk score were screened out. Among them, the high score group was mainly related to drug resistance, and the low score group was mainly related to drug sensitivity (Fig.5G).

\section{Discussion}

M6A RNA methylation modification is involved in almost all stages of RNA regulation, affecting RNA maturation, transcription, localization, translation and metabolism, thus playing an important biological function [19]. Studies [20,21] have shown that there are differences in the mRNA expression levels of m6A RNA methylation regulators between prostate cancer patients and normal samples. Among them, $50 \%$ of the m6A methylation recognition protein and methyltransferase are highly expressed in tumor samples, while FTO and ALKBH5 are low expressed in tumor samples. Multivariate Cox regression analysis showed that IGF2BP3, HNRNPA2B1, METTL14 and ALKBH5 were significantly correlated with the recurrence free survival rate of prostate cancer patients [20], indicating that the high level of m6A methylation in mRNA affects the subcellular localization of protein, promotes the progress of prostate cancer, and makes the survival benefit of patients poor. In terms of mutation, we found that the overall mutation rate of $\mathrm{m} 6 \mathrm{~A}$ was $8.58 \%$, and the highest mutation rate was $\mathrm{ZC} 3 \mathrm{H} 13$, which reached $21 \%$. Moreover, survival analysis found that the prognosis of the mutation group was significantly poor, which indicated that the mutation of m6A could affect the prognosis of prostate cancer. In addition, we found that m6A related mutations were more enriched in RTK signaling pathway by gsva analysis. Strict regulation of RTK activity controls the most basic processes of cells, such as cell cycle, proliferation, differentiation, movement, cell death and survival [21], which indicates that m6A related genes may affect the progress of prostate cancer through RTK related pathway. We found that the corresponding expression of m6A was increased and decreased in the follow-up copy number amplification. The change of CNV could not fully explain the difference in the expression of m6A between tumor and normal tissue, indicating that the progression of prostate cancer is a complex process, and other factors (such as methylation and transcription factors) also regulate the expression.

We clustered all samples according to the expression of m6A, and got two groups (Cluster_1 and cluster_2) with significant difference in prognosis. The GO of up-regulated genes between two groups were mainly enriched in stone modification and covalent chromotin modification, and the KEGG was mainly enriched in proteoglycans in cancer and focal adhesion. The Go of down-regulated genes was mainly enriched in oxidative phosphorylation, and KEGG is mainly enriched in thermogenesis and oxidative phosphorylation, respectively. Then we identified two distinct RNA modification patterns based on the expression of 3022 DEGs from two m6A clusters, and constructed a scoring model using genes with prognostic value, which is a poor and independent prognostic indicator to evaluate the prognosis of 
patients. Cancer related pathways suggested that high score group was positively correlated with RTK and RasMAPK, and negatively correlated with Cellcycle, EMT and hormone Er. Abnormal expression or activation of RTK in prostate cancer tissue, or increased expression of some RTK during castration, such as EGFR, PDGFR, FGFR, VEGFR, etc., lead to continuous activation of downstream signaling pathways, leading to proliferation and metastasis of tumor cells [22]. This may be the reasons for the poor prognosis of PFS in risk_score-high group. In addition, in-depth study of cell growth signaling pathway found that prostate cancer cells did not rely on androgen, but activated androgen receptor (AR) activity through PTK signaling pathway [23]. RTK may be an important part of the compensation mechanism of AR activation under androgen deprivation. At least part of RTK may play a role in the formation of hormone resistant prostate cancer by directly regulating AR signaling pathway.

At present, studies have shown that PD-1 and PD-L1 have certain expression and related role in prostate cancer[10,24]. Sfanos et al. [24] found that PD-1 was overexpressed in CD8 ${ }^{+} \mathrm{T}$ lymphocytes in prostate cancer tissues, resulting in the failure of these $\mathrm{CD} 8^{+} \mathrm{T}$ lymphocytes to produce corresponding anti-cancer response. Animal studies also show that the efficacy and prognosis of immunotherapy for prostate cancer are related to the expression of PD-1/PD-L1 on related cells and the combination of PD-1/PD-L1 inhibitors can significantly prolong the progression free survival of experimental animals[25]. Our heat map of checkpoint related genes showed that checkpoint genes were highly expressed in high score group. SIGLEC15, TIGIT, CD274, HAVCR2, LAG3 and PDCD1LG2 were highly expressed in high risk group. Using risk score to predict patients' response to ICB treatment, we found that low risk score patients had significant clinical benefits, which further indicated that risk stratification of prostate cancer can guide ICB treatment and prognosis evaluation.

The response of immunotherapy usually depends on the interaction between tumor cells and immune regulation in TME. Tumor infiltrating lymphocytes (TILs) are tumor antigen induced lymphocytes, which play an important role in immune surveillance and killing cancer cells in TME. TILs mainly include tumor infiltrating T lymphocytes, natural killer cells and tumor infiltrating B lymphocytes $[26,27]$. The correlation between tumor TIL level and prognosis shows the positive role of immune monitoring. When the number and proportion of $\mathrm{CD} 4^{+} \mathrm{T}$ cell components remain stable in TME, it can enhance the body's immunity. Studies have shown that the number of $\mathrm{CD} 8^{+} \mathrm{T}$ cells in lung cancer microenvironment can be used as an independent prognostic factor [28]. NK cells infiltrated in TME also play an important role in inhibiting tumor growth and metastasis [29]. We found that the infiltration of B cells, T cells CD $4^{+}$, endothelial cells and $\mathrm{T}$ cells $\mathrm{CD} 8^{+}$was higher in low score group , and the higher the risk score, the more obvious the response to ICB treatment. In addition, the high score group was mainly related to drug resistance, and the low score group was mainly related to drug sensitivity. The patients in risk_ score-low have obvious clinical benefit, which may be the reason of TME with better prognosis in risk_score-low.

Genetic instability, such as MSI, may be an important molecular event leading to tumorigenesis and development [30]. MSI molecular phenotype is not common in prostate cancer, and has significance in anti-PD-1 / PD-L1 therapy [31]. In 2017, the U.S. Food and Drug Administration approved pembrolizumab 
for the treatment of solid tumors containing MSI. Microsatellite instability accounted for about $3 \%$ of patients with metastatic castration resistant prostate cancer [32], but not all patients with MSI phenotype responded to PD-1 / PD-L1 treatment. In our study, the MSI score of risk_score-low group was significantly higher than that of risk_score-high group, and its response to ICB treatment was higher than that of high score group. We also found that MSI showed a new possibility of ICB treatment in different immune types and immune microenvironment. TME with B cells, T cells $\mathrm{CD} 4^{+}$and $\mathrm{T}$ cells $\mathrm{CD} 8^{+}$was significantly lower in risk_score-high group, it can be concluded that the risk score model can well reflect the tumor immune infiltration pattern and guide immunotherapy.

\section{Conclusion}

We explored the interaction between various m6A RNA modifications on the adenylate of prostate cancer, and used this interaction to characterize the tumor microenvironment and pharmacogenomic map, and concluded that their interaction mechanism can affect the tumor microenvironment and related to the prognosis of prostate cancer. Our risk score model reflect the regulatory role of m6A in transcription, and identified the role of the risk score model in tumor microenvironment and immunotherapy. In a word, it provides a new idea and possibility to improve the individualized immunotherapy of prostate cancer by identifying different immunophenotypes of tumor.

\section{Abbreviations}

N6,2'-0-dimethyladenosine: m6A;

prostate carcinoma: PCA;

principal component analysis: PCA;

the least absolute shrinkage and selection operator: LASSO;

receiver operating characteristic: ROC;

overall survival: OS;

Progression-free survival:PFS;

The Cancer Genome Atlas: TCGA;

tumor microenvironment $\rrbracket$ TME

differentially expressed genes $\triangle \mathrm{DEGs} \bigotimes$

cytotoxic T-lymphocyte antigen $4 \llbracket C T L A-4) \rrbracket$

programmed death $1 /$ programmed death ligand 1 1 PD-1/PD-L1区 
lymphocyte activation gene $3 \varangle \mathrm{LAG}-3 \rrbracket$

Immune Checkpoint Blockade『ICB囚

the Tumor Immune Dysfunction and Exclusion $₫ T$ IDE $\$

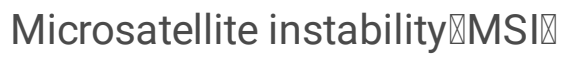

cancer therapeutics response portal『CTRP

\section{Declarations}

\section{Acknowledgment}

Not applicable.

\section{Authors' contributions}

JQH, SL, XDL and XQL conceived and designed the study, which were proofed by XDL and XQL. SL, SRW YFH and JZA analyzed the data and wrote the manuscript. All authors read and approved the final manuscript.

\section{Funding}

The study was funded by the Key R \& D and promotion projects of Henan Province in 2021 (Tackling key scientific and technological problems, Grant no. 212102310685 and 202102310429), the Construction project for improving the ability of diagnosis and treatment of difficult diseases(Grant no.ZLYNXM202009) and the science and technology public relations program of Henan Province(Grant no.LHGJ20190541).

\section{Availability of data and materials}

The datasets that support the findings of this study are available from the corresponding author upon reasonable request.

\section{Ethics approval and consent to participate}

This study was approved by the Ethics Committee of Henan University. All participants gave written, informed consent.

\section{Consent for publication}

Not applicable.

\section{Conflicts of interest}

The authors state that they have no conflicts of interest. 


\section{References}

1. Roignant JY, Soller M. m6A in mRNA: An Ancient Mechanism for Fine-Tuning Gene Expression. Trends Genet. 2017 ;33(6):380-390.

2. Ou-Yang S, Liu JH, Wang QZ. Expression patterns and a prognostic model of m6A-associated regulators in prostate adenocarcinoma. Biomark Med. $2020 ; 14(17): 1663-1677$.

3. Schlessinger J. Cell signaling by receptor tyrosine kinases. Cell. 2000;103(2):211-25.

4. Nagai A, Watanabe M, Nasu Y, Iguchi H, Kusumi N, Kumon H. Analysis of human ejaculation using color Doppler ultrasonography: a comparison between antegrade and retrograde ejaculation. Urology. 2005;65(2):365-8.

5. Chen Y, Xin X, Li J, Xu J, Yu X, Li T, Mo Z, Hu Y. RTK/ERK pathway under natural selection associated with prostate cancer. PLoS One. 2013;8(11):e78254.

6. Sfanos KS, Bruno TC, Meeker AK, De Marzo AM, Isaacs WB, Drake CG. Human prostate-infiltrating CD8+ T lymphocytes are oligoclonal and PD-1+. Prostate. 2009;69(15):1694-703.

7. Rekoske BT, Olson BM, McNeel DG. Antitumor vaccination of prostate cancer patients elicits PD1/PD-L1 regulated antigen-specific immune responses. Oncoimmunology. 2016;5(6):e1165377.

8. Ye L, Zhang F, Li H, Yang L, Lv T, Gu W, Song Y. Circulating Tumor Cells Were Associated with the Number of T Lymphocyte Subsets and NK Cells in Peripheral Blood in Advanced Non-Small-Cell Lung Cancer. Dis Markers. 2017;2017:5727815.

9. Wu T, Dai Y. Tumor microenvironment and therapeutic response. Cancer Lett. 2017 ;387:61-68.

10. Huang LJ, Deng XF, Chang F, Wu XL, Wu Y, Diao QZ. Prognostic significance of programmed cell death ligand 1 expression in patients with ovarian carcinoma: A systematic review and metaanalysis. Medicine (Baltimore). 2018;97(43):e12858.

11. Gong C, Yao C, Xu Z, Ni Z, Zhu X, Wang L, Yan X, Zhou W, Zhu S. Enhancement of NK cell-mediated lysis of non-small lung cancer cells by nPKC activator, ingenol 3,20 dibenzoate. Mol Immunol. 2017 ;83:23-32.

12. Hause RJ, Pritchard CC, Shendure J, Salipante SJ. Classification and characterization of microsatellite instability across 18 cancer types. Nat Med. 2016;22(11):1342-1350.

13. Abida W, Cheng ML, Armenia J, Middha S, Autio KA, Vargas HA, Rathkopf D, Morris MJ, Danila DC, Slovin SF, Carbone E, Barnett ES, Hullings M, Hechtman JF, Zehir A, Shia J, Jonsson P, Stadler ZK, Srinivasan P, Laudone VP, Reuter V, Wolchok JD, Socci ND, Taylor BS, Berger MF, Kantoff PW, Sawyers CL, Schultz N, Solit DB, Gopalan A, Scher HI. Analysis of the Prevalence of Microsatellite Instability in Prostate Cancer and Response to Immune Checkpoint Blockade. JAMA Oncol. 2019;5(4):471-478.

14. Reichert ZR, Urrutia J, Alumkal JJ. Microsatellite Instability as an Emerging Biomarker for Checkpoint Inhibitor Response in Advanced Prostate Cancer. JAMA Oncol. 2019;5(4):478-479.

15. Gao J, Aksoy BA, Dogrusoz U, Dresdner G, Gross B, Sumer SO, Sun Y, Jacobsen A, Sinha R, Larsson E, Cerami E, Sander C, Schultz N. Integrative analysis of complex cancer genomics and clinical profiles using the cBioPortal. Sci Signal. 2013;6(269):pl1 . 
16. Jiang P, Gu S, Pan D, Fu J, Sahu A, Hu X, Li Z, Traugh N, Bu X, Li B, Liu J, Freeman GJ, Brown MA, Wucherpfennig KW, Liu XS. Signatures of T cell dysfunction and exclusion predict cancer immunotherapy response. Nat Med. 2018;24(10):1550-1558.

17. Hu W, Wang G, Chen Y, Yarmus LB, Liu B, Wan Y. Coupled immune stratification and identification of therapeutic candidates in patients with lung adenocarcinoma. Aging (Albany NY). 2020;12(16):16514-16538.

18. Rees MG, Seashore-Ludlow B, Cheah JH, Adams DJ, Price EV, Gill S, Javaid S, Coletti ME, Jones VL, Bodycombe NE, Soule CK, Alexander B, Li A, Montgomery P, Kotz JD, Hon CS, Munoz B, Liefeld T, Dančík V, Haber DA, Clish CB, Bittker JA, Palmer M, Wagner BK, Clemons PA, Shamji AF, Schreiber SL. Correlating chemical sensitivity and basal gene expression reveals mechanism of action. Nat Chem Biol. 2016;12(2):109-16.

19. Siegel RL, Miller KD, Jemal A. Cancer statistics, 2020. CA Cancer J Clin. 2020 ;70(1):7-30.

20. Mansinho A, Macedo D, Fernandes I, Costa L. Castration-Resistant Prostate Cancer: Mechanisms, Targets and Treatment. Adv Exp Med Biol. 2018;1096:117-133.

21. Krug RM, Morgan MA, Shatkin AJ. Influenza viral mRNA contains internal N6-methyladenosine and 5'-terminal 7-methylguanosine in cap structures. J Virol. 1976 ;20(1):45-53.

22. Meyer KD, Saletore Y, Zumbo P, Elemento O, Mason CE, Jaffrey SR. Comprehensive analysis of mRNA methylation reveals enrichment in 3' UTRs and near stop codons. Cell. $2012 ; 149(7): 1635-46$.

23. Zhao BS, Roundtree IA, He C. Post-transcriptional gene regulation by mRNA modifications. Nat Rev Mol Cell Biol. 2017 Jan;18(1):31-42. doi: 10.1038/nrm.2016.132. Epub 2016 Nov 3. Erratum in: Nat Rev Mol Cell Biol. 2018;19(12):808.

24. Dyck L, Mills KHG. Immune checkpoints and their inhibition in cancer and infectious diseases. Eur J Immunol 2017;47(5): 765-779.

25. Xia L, Liu Y, Wang Y. PD-1/PD-L1 Blockade Therapy in Advanced Non-Small-Cell Lung Cancer: Current Status and Future Directions. Oncologist 2019; 24(1): 31-41.

26. Haanen JB, Robert C. Immune Checkpoint Inhibitors. Prog Tumor Res 2015; 42(2015): 55-66.

27. Cui SS, Du JJ, Liu SH, et al. Serum soluble lymphocyte activation gene-3 as a diagnostic biomarker in Parkinson's disease: A pilot multicenter study. Mov Disord 2019; 34(1): 138-141.

28. Sharma P, Allison JP. The future of immune checkpoint therapy. Science. 2015. 348(6230): 56-61.

29. Hoos A. Development of immuno-oncology drugs - from CTLA4 to PD1 to the next generations. Nat Rev Drug Discov. 2016. 15(4): 235-47.

30. Beer TM, Kwon ED, Drake CG, Fizazi K, Logothetis C, Gravis G, Ganju V, Polikoff J, Saad F, Humanski P, Piulats JM, Gonzalez Mella P, Ng SS, Jaeger D, Parnis FX, Franke FA, Puente J, Carvajal R, Sengeløv L, McHenry MB, Varma A, van den Eertwegh AJ, Gerritsen W. Randomized, Double-Blind, Phase III Trial of Ipilimumab Versus Placebo in Asymptomatic or Minimally Symptomatic Patients With Metastatic Chemotherapy-Naive Castration-Resistant Prostate Cancer. J Clin Oncol. 2017;35(1):4047. 
31. Topalian SL, Hodi FS, Brahmer JR, Gettinger SN, Smith DC, McDermott DF, Powderly JD, Carvajal RD, Sosman JA, Atkins MB, Leming PD, Spigel DR, Antonia SJ, Horn L, Drake CG, Pardoll DM, Chen L, Sharfman WH, Anders RA, Taube JM, McMiller TL, Xu H, Korman AJ, Jure-Kunkel M, Agrawal S, McDonald D, Kollia GD, Gupta A, Wigginton JM, Sznol M. Safety, activity, and immune correlates of anti-PD-1 antibody in cancer. N Engl J Med. 2012;366(26):2443-54.

32. Zheng W, Dong X, Zhao Y, Wang S, Jiang H, Zhang M, Zheng X, Gu M. Multiple Functions and Mechanisms Underlying the Role of METTL3 in Human Cancers. Front Oncol. 201912;9:1403.

\section{Tables}

Table 1. Demographic and clinicopathological characteristics of patients with PCA in TCGA database 


\begin{tabular}{|ll|}
\hline $\begin{array}{l}\text { Demographic or } \\
\text { Characteristics }\end{array}$ & $\begin{array}{l}\text { All subjects } \\
(\mathrm{N}=452)(\%)\end{array}$ \\
\hline Age at diagnosis & \\
\hline У=49 & $21(4.6)$ \\
\hline $50-59$ & $152(33.6)$ \\
\hline $60-69$ & $233(25.2)$ \\
\hline $70-79$ & $46(51.6)$ \\
\hline$>=80$ & $0(0.0)$ \\
\hline Stage_M & \\
\hline Female & $440(97.3)$ \\
\hline Male & $12(2.7)$ \\
\hline Stage_T & \\
\hline I & $5(1.1)$ \\
\hline II & $162(35.8)$ \\
\hline III & $274(60.6)$ \\
\hline IV & $11(2.5)$ \\
\hline Iymphatic_invasion & \\
\hline NO & $373(82.5)$ \\
\hline YES & $79(17.5)$ \\
\hline Risk score & $220(48.7)$ \\
\hline High & $232(51.3)$ \\
\hline Low & \\
\hline
\end{tabular}

Figures 


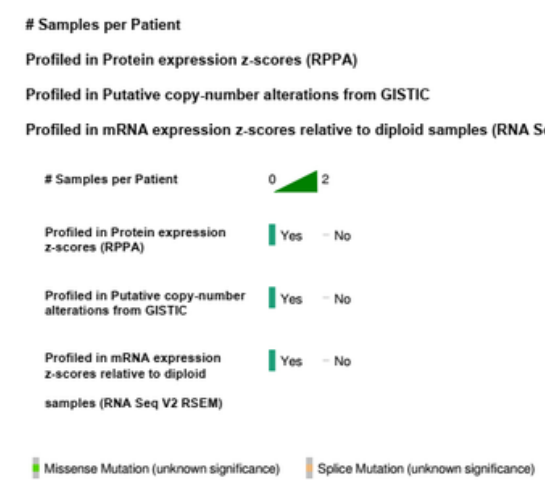

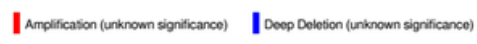

if Truncating Mutation (unknown sigriscance)

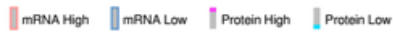

| No alloratoons

\section{B}

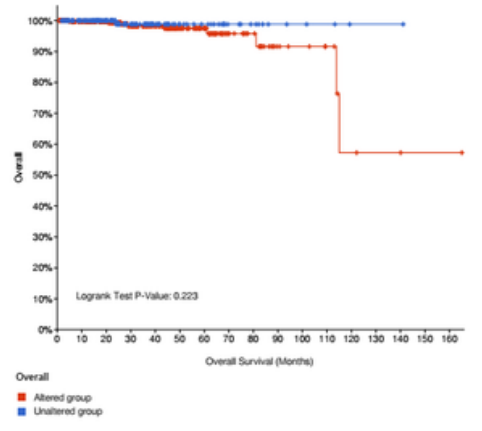

|

1.

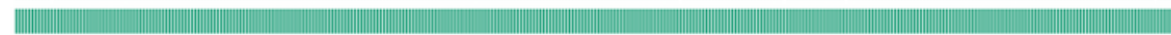

5\% |||

18\%

IGF2BP2 $7 \%$ | || ||

HNRNPA2B16\% ||| || ||| || |||||||||||| | || || ||

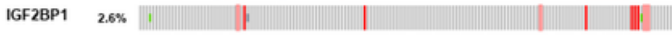

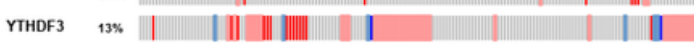

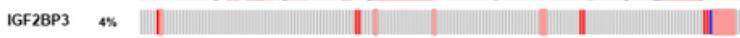

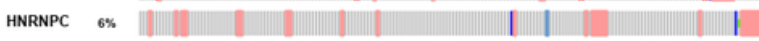

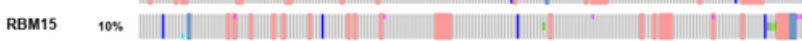

RBMx $7 \%$ |

METL14 10\% |

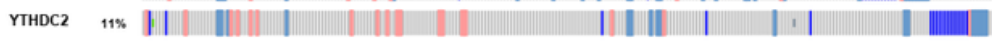

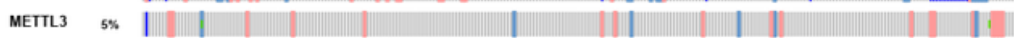

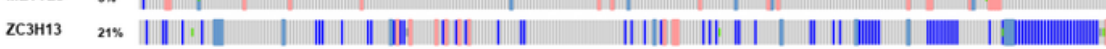

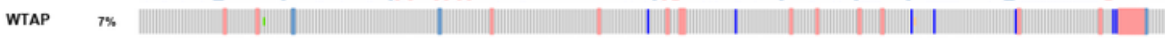

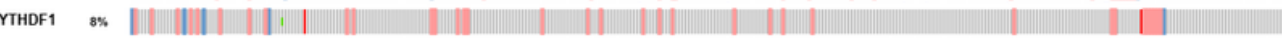

Үтнос1 \%

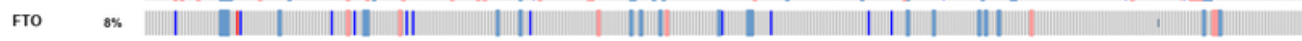

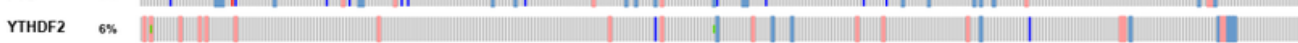

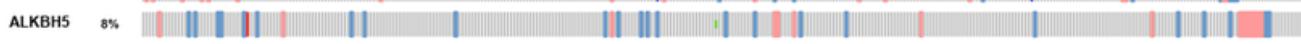
Genetic Alteration
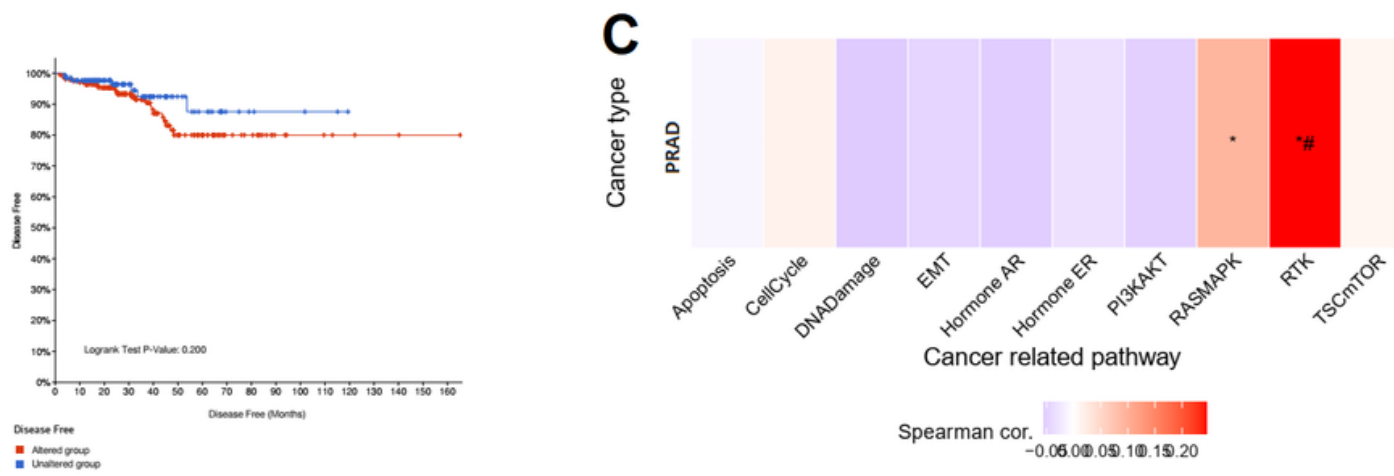

Figure 1

Mutation analysis of m6A modification related genes. In 452 samples, 39 (8.58\%) of m6A had RNA modification mutations, among which $\mathrm{ZC} 3 \mathrm{H} 13$ had the highest mutation rate, reaching $21 \%(\mathrm{~A})$. The OS and PFS of the mutation group was significantly lower than that of the non mutation group $(P=0.021)$ (B). GSVA showed that the mutation group of m6A gene was more enriched in RTK and EASMAPK signaling pathway $(\mathrm{C})$. 


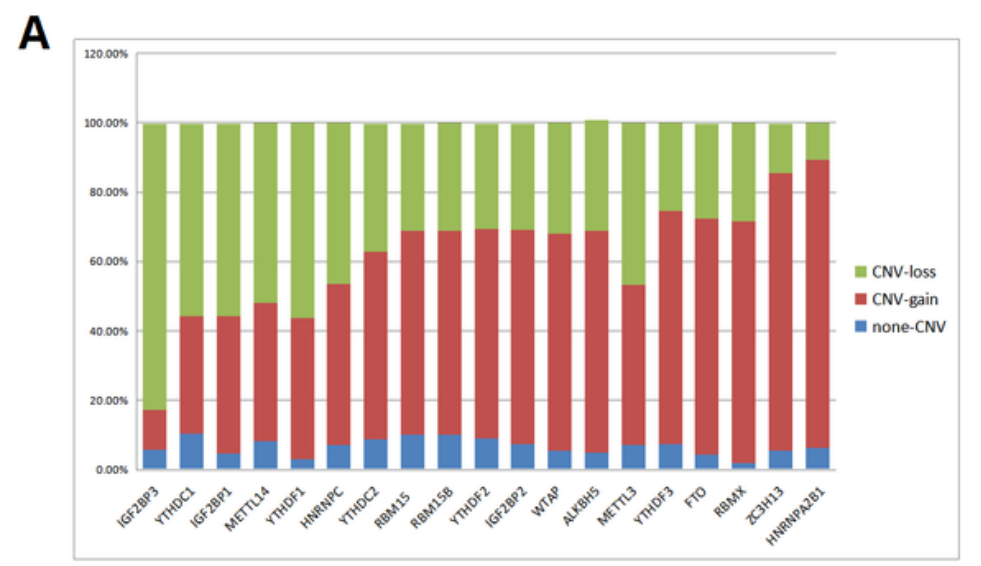

B
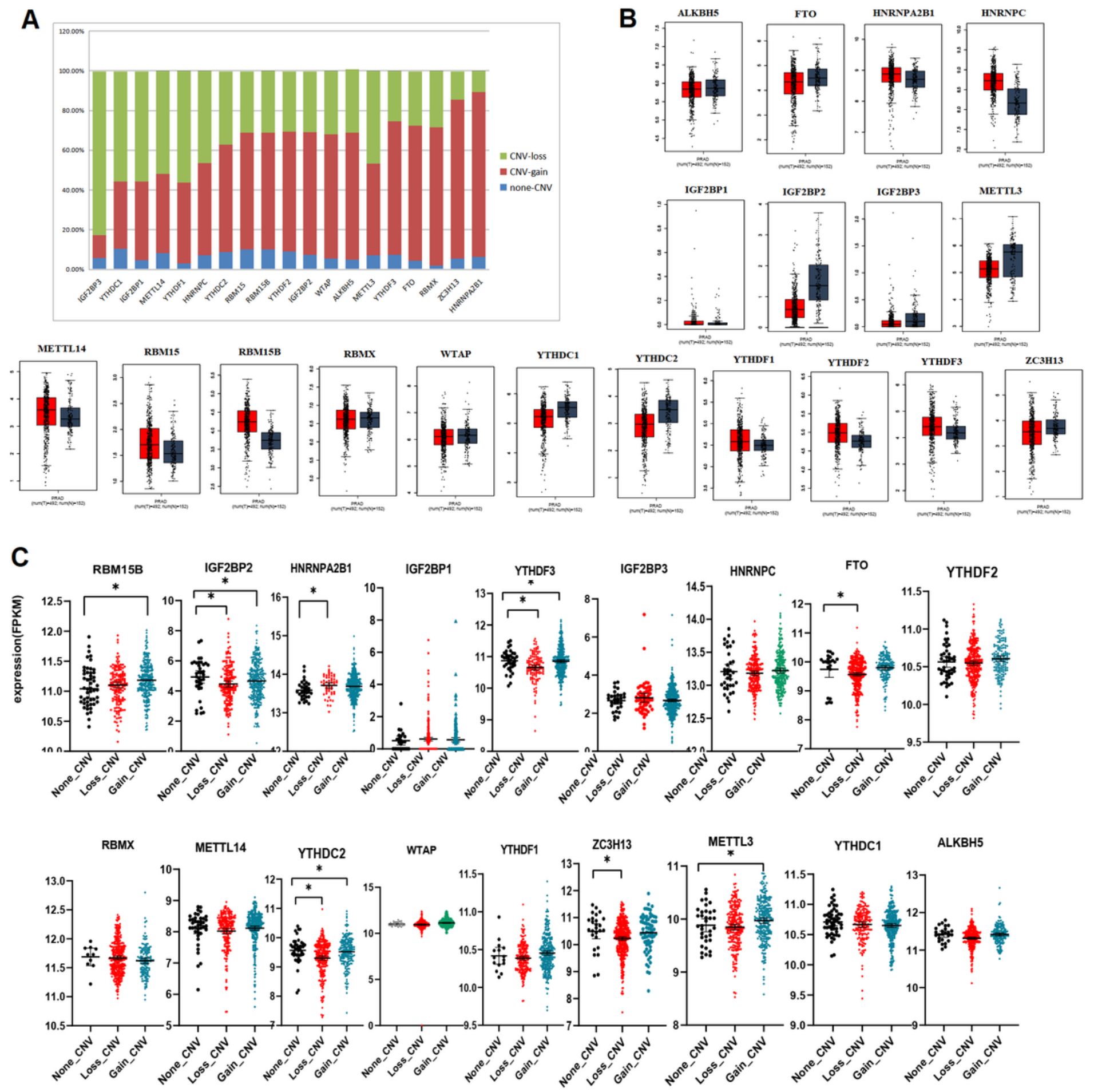

Figure 2

Copy number analysis of genes related to m6A modification. Figure A shows the change of copy number of each kind of m6A, and Figure B shows the expression of each kind of m6A in tumor and paired normal. The samples were divided into three groups (CNV gain, CNV loss, none CNV) according to the CNV of m6A modified gene, and the expression of m6A modified gene in these three groups was compared (C). 
A
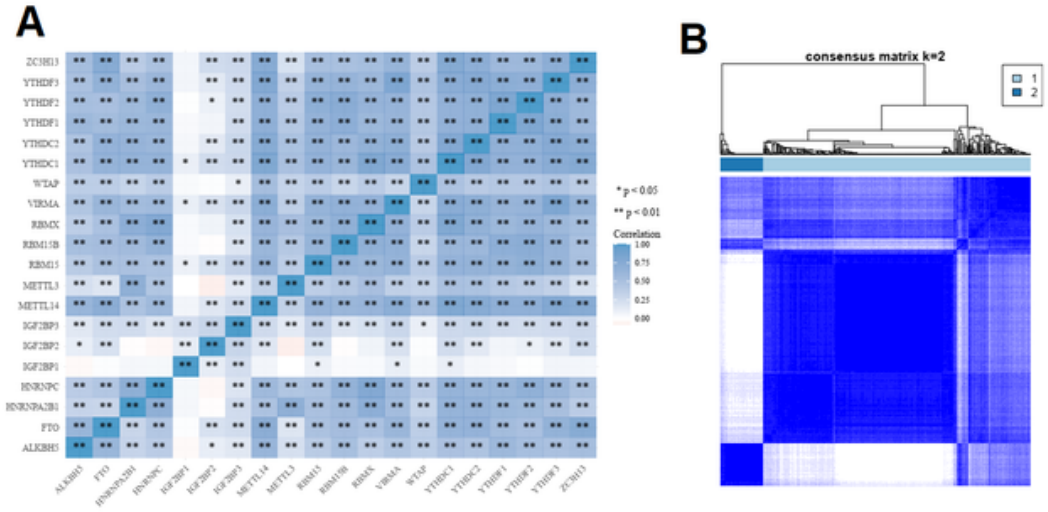

D
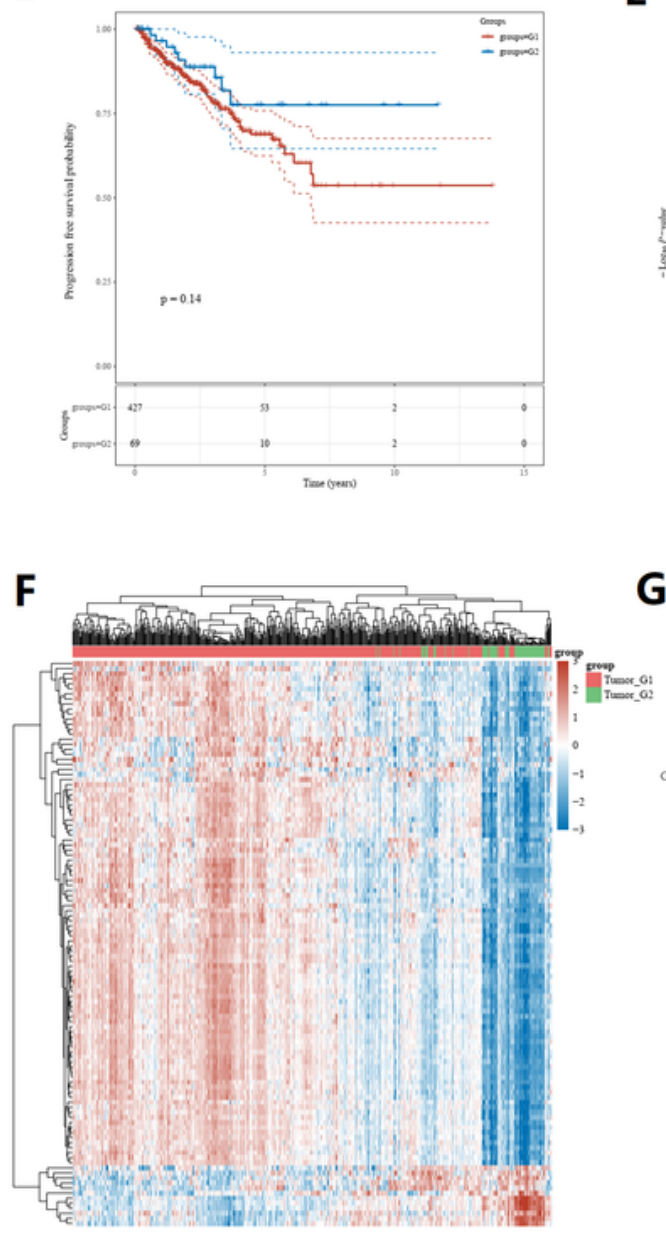

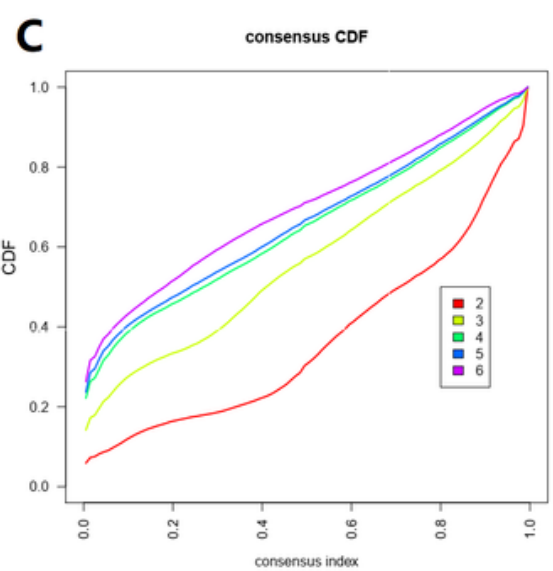

$\mathbf{E}$

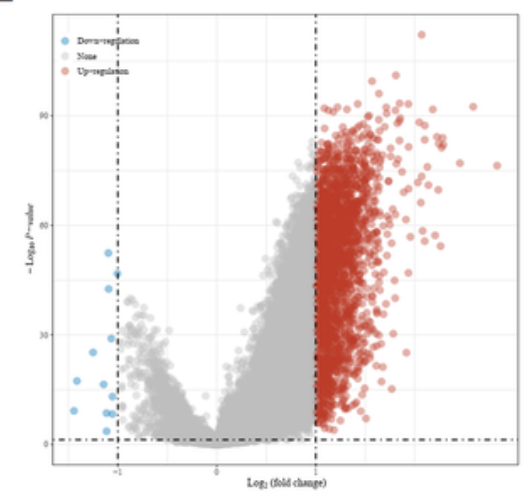

$\mathbf{G}$
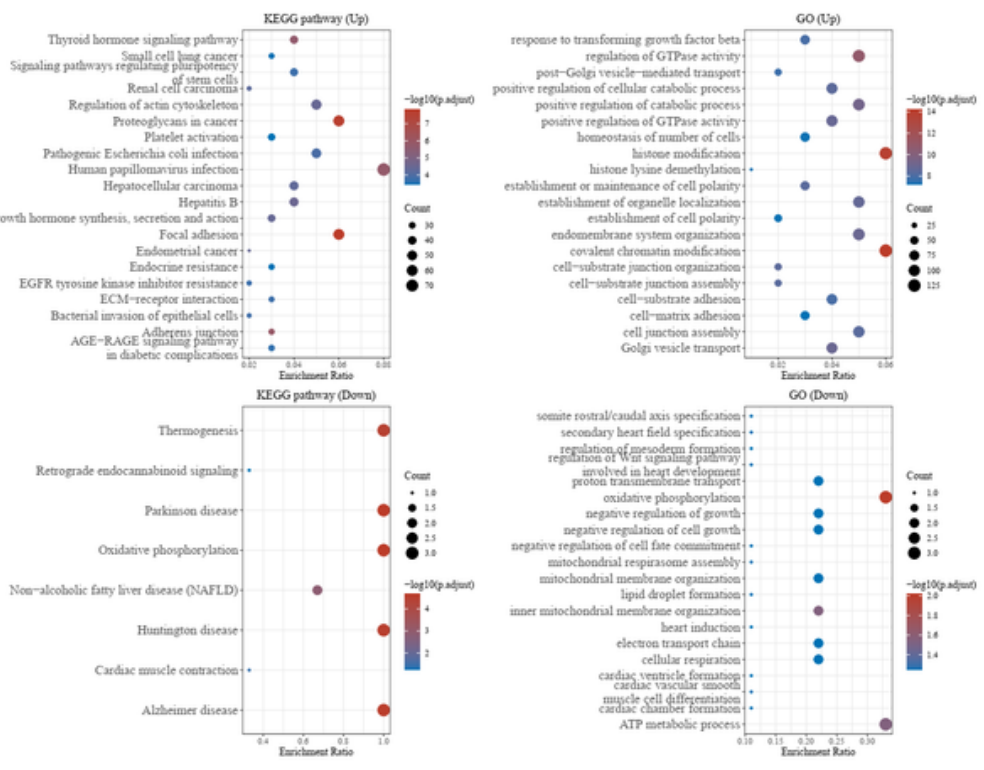

\section{Figure 3}

Different RNA modification patterns associated with hallmark in cancer. There was interaction among 20 m6A modified gene tables (A). All samples were clustered according to the expression of these $20 \mathrm{~m} 6 \mathrm{~A}$ modified genes, and two clusters were obtained (Cluster_ 1 and cluster_ 2$)(B, C)$. The prognosis of cluster 2 was better than that of cluster 1 (D). The gene expression heat map and volcano map (E, F) of two 
clusters were drawn, and the GO and KEGG enrichment analysis (g) of these two types of differential genes were carried out $(\mathrm{G})$.
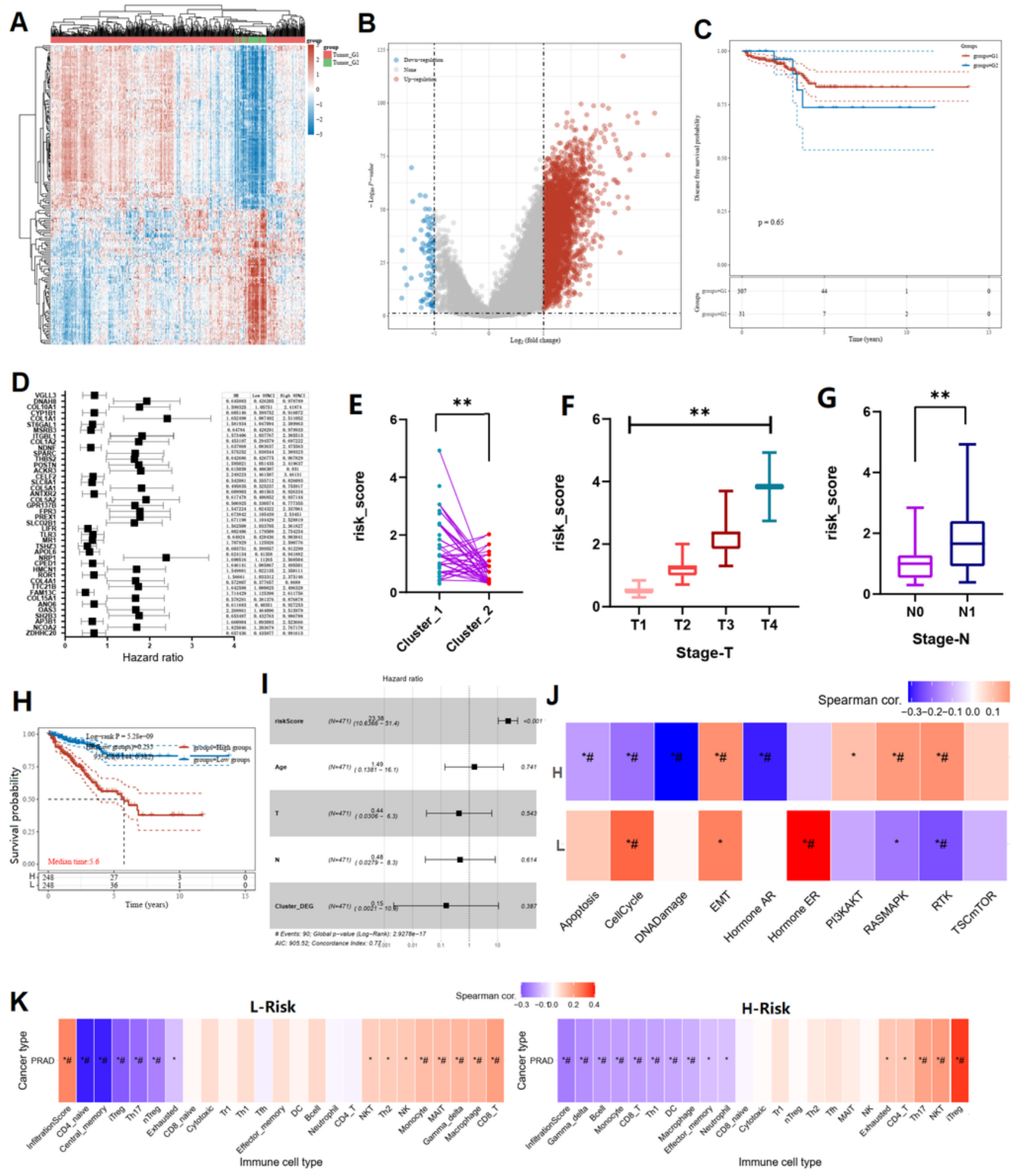

Figure 4

Signature of m6A RNA modification. The 3022 differentially expressed genes from PCA transcripts were divided into two groups by unsupervised clustering (gene. Cluster_A and gene. Cluster_B), and the maps of hot earth and volcano were drawn $(A, B)$. The prognosis of gene_Cluster $A$ was worse than that of 
gene_Cluster B (C). Univariate prognostic analysis (D) was performed for 3022 differentially expressed genes. The risk score in Cluster_1group was significantly higher than that in group 2 (E). The PFS of low risk_score group was higher than that in high group $(\mathrm{H})$. Multivariate Cox regression analysis of risk_score is a poor and independent prognostic index (I). Cancer related pathways (GSVA) analysis showed that in high score group, there was a negative correlation with Cell cycle, EMT and hormone Er, and a positive correlation with RTK, EMT and RasMAPK, while the result of low score group was opposite to that of the control group $(\mathrm{J})$. The immune correlation between high- and low-score group was analyzed by GSVA (K). 
A
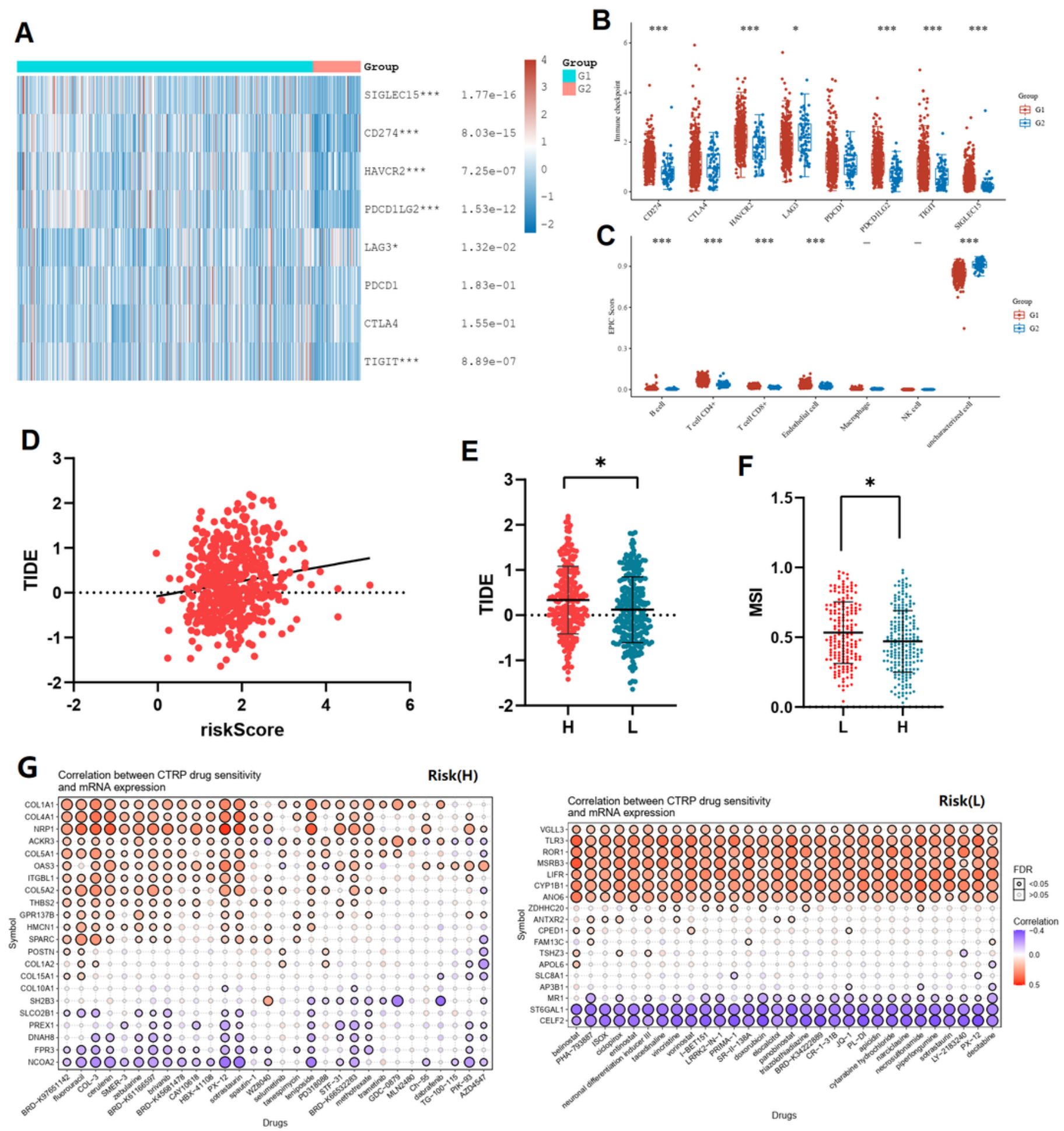

Figure 5

Prediction of immune microenvironment and immunotherapy response by risk score model. Heat map of immune checkpoint related genes showed that immune checkpoint genes were highly expressed in high risk group (A). The histograms showed that SIGLEC15, TIGIT, CD274, HAVCR2, LAG3and PDCD1LG2 were highly expressed in high risk group (B). The proportion of all kinds of immune cells in high- and low- score groups was made by CIBERSORT method (C). The risk score was positively correlated with the TIDE score 
(D). The TIDE score of the high risk group was higher than that of the low risk group (E). The MSI score of the high risk group was significantly higher than that of the low risk group $(F)$. The relationship between risk score and drug response of cancer cell lines in CTRP database (G). 